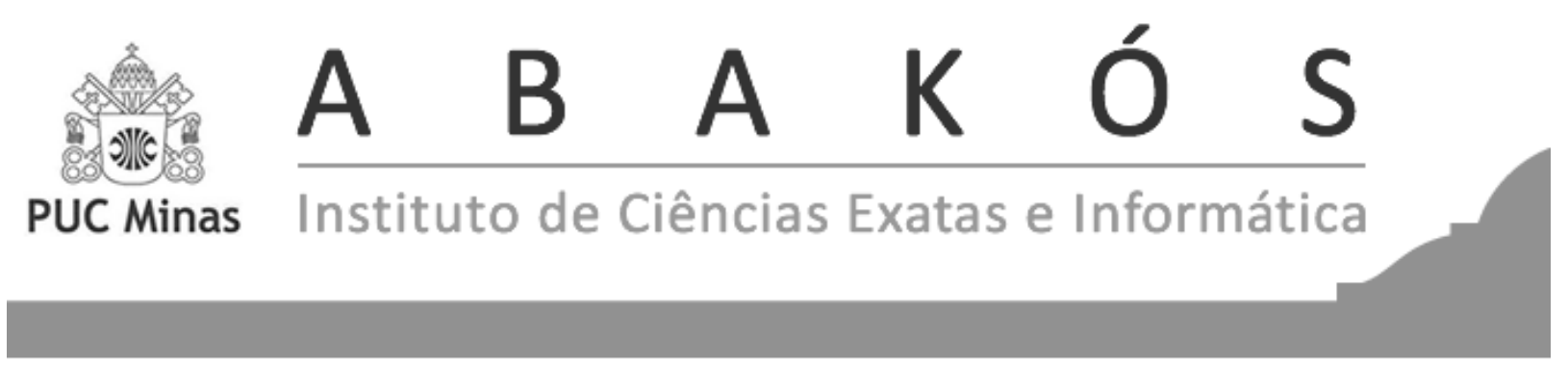

\title{
Heurísticas de Usabilidade para Aplicativos Móveis Educacionais Infantis*
}

\author{
Usability Heuristics for Children Educational Mobile App
}

\author{
Caio Almeida Abreu ${ }^{1}$ \\ Jean C. S. Rosa ${ }^{2}$ \\ Ecivaldo de Souza Matos ${ }^{3}$
}

\begin{abstract}
Resumo
O mercado de aplicativos móveis educacionais apresenta uma gama diversificada de opções destinadas à satisfação de diversos públicos, sobretudo o infantil. Todavia, parte considerável desses aplicativos abrange diversas idades e desconsidera características intrínsecas à educação infantil, em que crianças têm necessidades de interação diferenciadas e necessidades educacionais específicas. No campo dos estudos de informática na educação, encontrou-se apenas um conjunto de heurísticas de usabilidade para aplicativos móveis educacionais (heurísticas genéricas); todavia, essas heurísticas não consideram aspectos específicos da educação infantil. Nesse sentido, este artigo apresenta os resultados de uma pesquisa em que foi desenvolvido e avaliado um conjunto de heurísticas de usabilidade para aplicativos móveis educacionais específicos para o domínio infantil. As novas heurísticas foram avaliadas por professores e pesquisadores da Educação Infantil, especialistas em Interação Humano-Computador e pesquisadores em Informática na Educação. Além disso, a eficácia e a eficiência dessas novas heurísticas foram avaliadas em relação às heurísticas genéricas, por meio de um experimento no qual identificou-se o grau de cobertura das novas heurísticas, as quais se mostraram mais adequadas para avaliação de aplicativos móveis educacionais quando no domínio infantil.
\end{abstract}

Palavras-chave: Usabilidade Pedagógica. Usabilidade Técnica. Avaliação Heurística. Interação Criança-Computador. m-learning .

\footnotetext{
* submetido em 06/03/2019 e aceito em 04/09/2020.

${ }^{1}$ Bacharel em Engenharia da Computação, Brasil- caioabreu@ outlook.com

${ }^{2}$ Doutorando em Ciência da Computação (DCC/UFBA), mestre em Ciência da Computação (DCC/UFBA) e tecnólogo em Sistemas para Internet (UNIT), Brasil- jean.rosa@ufba.br

${ }^{3}$ Professor e pesquisador do Departamento de Ciência da Computação (UFBA), do Centro de Estudos AfroOrientais (CEAO/UFBA) e do Programa de Pós-Graduação em Ensino, Filosofia e História das Ciências (UFBA/UEFS). Bolsista de Produtividade em Pesquisa do CNPq. Doutor em Educação (USP), mestre em Informática (UFCG) e bacharel em Ciência da Computação (UFBA), Brasil- ecivaldo@ufba.br
} 


\begin{abstract}
The market for educational mobile applications presents a diverse range of options aimed at different audiences' satisfaction, especially children. However, a considerable portion of these applications comprises multiple ages and disregards aspects intrinsic to early childhood education, where children have differentiated interaction needs and specific educational needs. In the field of computers in education studies, we found only a set of usability heuristics for educational mobile applications (generic heuristics); however, these heuristics do not consider specific aspects of early childhood education. In this sense, this paper presents the research results in which a set of usability heuristics for a specific educational app for children's domain was developed and evaluated. The new heuristics were evaluated by teachers and researchers of Child Education, specialists in HumanComputer Interaction, and researchers in Computing in Education. In addition, the efficacy and efficiency of the new heuristics evaluated considering generic heuristics, through an experiment in which the degree of coverage of the new heuristics was identified. The percentage of heuristics violated in the evaluation performed using generic heuristics was lower than the new heuristics' violations. Therefore, the new heuristics were more appropriate for evaluating educational mobile applications in the children's domain.

Keywords: Pedagogic Usability. Technical Usability. Heuristic Evaluation. ChildComputer Interaction. m-learning.
\end{abstract}




\section{INTRODUÇÃO}

A utilização de aplicativos móveis educacionais tem crescido e aberto espaço para o mobile learning (m-learning), termo utilizado nos estudos que relacionam mobilidade, aprendizagem e tecnologias móveis (SCHUCK et al., 2017). De acordo com Winckler e Pimenta (2002), a escolha de software de apoio ao ensino e à aprendizagem não deve se pautar por metodologias para uma população-alvo genérica, para aprendizagens apenas individuais, controle dos resultados da avaliação, dentre outros, pois não serão precisos devido à ausência do contexto de de outras variáveis, como tipo de aplicação em questão e perfil dos usuários.

Além desses fatores pedagógicos, fatores técnicos, como a usabilidade, devem ser considerados durante a concepção de software educacional (SQUIRES; PREECE, 1996). A usabilidade, enquanto categoria de qualidade de Interação Humano-Computador (IHC), é indispensável nesse tipo de software (MATOS, 2013). Para Squires e Preece (1996), a usabilidade é um dos fatores determinantes para o sucesso do ensino e da aprendizagem mediatizados por software.

Usabilidade, segundo Nielsen (1994), está intrinsecamente associada aos seguintes atributos: satisfação do usuário, facilidade e memorização da utilização do software, tratamento de erros e a eficiência no uso software. Nielsen apresentou 10 heurísticas de usabilidade e indicou a necessidade de adaptações nas heurísticas propostas para atender sistemas de domínios diferentes. Desse modo, nota-se a preocupação em a usabilidade considerar diferentes domínios de software e diferentes perfis de usuários.

Por meio de uma revisão sistemática da literatura, Gonçalves et al. (2011) evidenciaram que o modo para avaliar os diferentes tipos de softwares e plataformas devem seguir um padrão próprio, sobretudo software para dispositivos móveis. Para os autores, métodos ou heurísticas tradicionais de inspeção de usabilidade não se aplicam a software para dispositivos móveis, haja vista que o modo de interação e o tamanho de tela são diferentes.

Ao tratarem de dispositivos móveis, Bonifácio et al. (2010a) reiteram a importância da usabilidade, tendo em vista que um dos seus objetivos é minimizar a dificuldade de interação dos usuários, o que pode resultar na melhoria da qualidade do software. Bonifácio et al. (2010b) destacam, assim como Nielsen (1994), que as heurísticas de usabilidade devem ser adequadas ao software que está sendo avaliado ou desenvolvido.

Todavia, há poucas produções científicas acerca da avaliação de aplicativos móveis educacionais por heurísticas de usabilidade. D'Carlo et al. (2016) enfatizam a escassez de publicações nesse domínio ao afirmarem que "no âmbito de trabalhos direcionados especificamente para aplicativos móveis educacionais não foram encontradas iniciativas similares" (p. 778). As autoras também afirmam que "não foram encontradas heurísticas que abordassem aspectos específicos de usabilidade para os diferentes domínios de aplicativos, sobretudo para os aplicativos educacionais" (D’CARLO et al., 2016, p. 775). A partir dessa evidência, despertou-se o interesse de investigar um domínio ainda mais específico, o domínio educacional infantil. A busca na literatura também indicou carência de heurísticas de usabilidade nesse domínio espe- 
cífico.

Nesse sentido, este artigo apresenta heurísticas de usabilidade para aplicativos educacionais móveis infantis (HE), sua avaliação inicial e resultados de um estudo experimental ${ }^{4}$ que compara essas novas heurísticas com as heurísticas de usabilidade para aplicativos educacionais móveis de D'Carlo et al. (2016) e D'Carlo et al. (2017), neste texto chamadas de heurísticas gerais (HG).

Este artigo está organizado em oito seções. A Seção 2 apresenta o conceito de usabilidade em softwares educacionais. A Seção 3 é destinada à apresentação de estudos relacionados a heurísticas de usabilidade para aplicativos móveis e para aplicativos móveis educacionais. A metodologia de pesquisa será apresentada na quarta seção. Os elementos caracterizadores do domínio infantil e as heurísticas de usabilidade para aplicativos móveis educacionais infantis serão apresentados nas Seções 5 e 6, respectivamente. A Seção 7 apresenta a análise dos resultados da avaliação das heurísticas, seguida das conclusões e trabalhos futuros na Seção 8.

\section{USABILIDADE EM SOFTWARES EDUCACIONAIS}

Software educacional possui a relevante tarefa de potencializar os processos de ensino e de aprendizagem por meio do diálogo entre o estudante e o professor midiatizado pela interface do usuário (MATOS, 2013). Por isso, é necessário que os educadores e os estudantes possam agir eficientemente sob a interface do software (GOMES; PADOVANI, 2005). Destarte, Matos (2013) aponta que novas formas de ensinar e de aprender surgiram e, portanto, o uso dos softwares educacionais deve convergir para a construção de conhecimentos dos estudantes.

Para a adoção de um software educacional é importante observar alguns critérios de qualidade, uma vez sua inadequação técnica ou pedagógica pode comprometer o desempenho didático-pedagógico. Dentre vários critérios, Piconez (2010) destaca a usabilidade como um aspecto essencial em software educacional. Para Coelho e Santoro (2002), a usabilidade é um dos fatores determinantes para o sucesso dos processos de ensino e de aprendizagem mediatizados por sistemas computacionais interativos. Todavia, conforme Gomes e Padovani (2005), a usabilidade de software educacional não deve se ater apenas a aspectos técnicos, mas deve valorizar também aspectos pedagógicos.

De acordo com Nielsen (1990), quando se trata de software educacional, há dois tipos de usabilidade: a usabilidade técnica e a usabilidade pedagógica. A usabilidade técnica é um subconceito de usabilidade, enquanto a usabilidade pedagógica é um subconceito de utilidade.

A usabilidade pedagógica está sujeita à satisfação de necessidades educacionais de estudantes e professores. De acordo com Nokelainen (2006), a usabilidade pedagógica de um sistema e/ou material de aprendizagem também depende de propósitos estabelecidos pelo estudante e pelo professor em situação de aprendizagem. Nesse contexto, a usabilidade pelo viés pedagógico aborda por meio da construção do conhecimento, uma perspectiva construtivista-

${ }^{4}$ Este artigo apresenta a expansão dos resultados evidenciados por Abreu et al. (2017a). 
interacionista, em que o estudante participa ativamente na construção do seu saber, tornando-se, por isso mesmo, mais responsável pela sua aprendizagem (PICONEZ, 2010).

Sob essa perspectiva, Nokelainen (2006) apresentou 10 heurísticas que caracterizam a avaliação da usabilidade pedagógica. Esse conjunto é composto pelos seguintes componentes: controle do aprendiz, atividade do aprendiz, aprendizagem cooperativa/colaborativa, orientação a objetivos, aplicabilidade, valor adicionado, motivação, valorização dos conhecimentos prévios, flexibilidade e feedback. Ainda segundo Nokelainen (2006), a usabilidade pedagógica deve prover um projeto do material de aprendizagem, cujas funções facilitem a aprendizagem desse material e sua distribuição.

A revisão sistemática da literatura realizada por Ávila et al. (2012) revela que ainda há poucos estudos sobre a usabilidade pedagógica. A compreensão da diferença entre os conceitos apresentados, sobretudo o da usabilidade pedagógica, viabilizou a adaptação dos critérios e heurísticas utilizadas nesta pesquisa ao meio educacional infantil, considerando características pedagógicas destinadas ao público-alvo.

\section{HEURÍSTICAS DE USABILIDADE PARA APLICATIVOS MÓVEIS EDUCACIONAIS}

Há diversos conjuntos de heurísticas de usabilidade para avaliação de software ( $c f$. (KNOLL, 2014; SALAZAR et al., 2012; ALMEIDA et al., 2013; BONIFÁCIO, 2012)). Entretanto, D'Carlo et al. (2016) apontaram a insuficiência de heurísticas que abordem aspectos específicos de usabilidade para os diferentes domínios de software, sobretudo para os aplicativos móveis educacionais. Essas autoras propuseram um conjunto de heurísticas para avaliar a usabilidade de aplicativos móveis educacionais, que chamaremos de Heurísticas Gerais (HG) neste texto, conforme a Tabela 1 .

\section{Tabela 1 - Heurísticas para aplicativos móveis educacionais}

\begin{tabular}{c|l} 
Cód. & \multicolumn{1}{|c}{ Descrição } \\
\hline HG1 & Consistência e bom aproveitamento do espaço da tela \\
HG2 & Disponibilidade (Acesso de qualquer lugar) e Portabilidade \\
HG3 & Facilidade de acesso às funcionalidades \\
HG4 & Resposta Imediata e Visibilidade \\
HG5 & Adequação de mensagem à funcionalidade e ao usuário \\
HG6 & Prevenção de erros e recuperação rápida ao último estado estável \\
HG7 & Aprendizado orientado a um objetivo \\
HG8 & Aprendizagem e Recordação no processo de Interação \\
HG9 & Controle de aprendizado e Flexibilidade \\
HG10 & Disponibilizar diferentes experimentações de aprendizado, incluindo erros \\
HG11 & Aprendizado cooperativo/colaborativo \\
HG12 & Motivação \\
HG13 & Consolidação de novos aprendizados (Aplicabilidade) \\
HG14 & Ajuda e documentação
\end{tabular}

Fonte: Retirado de D'Carlo et al. (2016).

Para a criação desse conjunto, as autoras consideraram três perspectivas: (1) heurísticas genéricas para aplicativos móveis, por descreverem requisitos básicos de usabilidade para a interface de qualquer tipo de aplicativo; (2) requisitos de usabilidade de sistemas educacionais 
apresentados por Nokelainen (2006) e Silveira e Carneiro (2012); e (3) o conhecimento de um especialista em educação. Além disso, D’Carlo et al. (2016) realizaram avaliações das heurísticas com especialistas em Interação Humano-Computador e Educação, com intuito de compreender sua relevância no contexto educacional, além de Avaliação Heurística e teste com usuários.

\section{METODOLOGIA}

Nesta seção apresentamos a metodologia aplicada na pesquisa. Inicialmente é apresentado como algumas heurísticas foram adaptadas para o uso em aplicativos móveis educacionais infantis e logo depois é mostrado como foi realizada a comparação das HE com as HG.

\subsection{Adaptação das Heurísticas}

A partir da necessidade de adaptação das heurísticas genéricas de usabilidade para contexto e domínios específicos apontada por Nielsen (1994), torna-se relevante que as características do contexto educacional infantil em aplicativos móveis não sejam ignoradas. Desse modo, esta pesquisa originou-se da evidência apontada por D'Carlo et al. (2016) quanto à carência de heurísticas de usabilidade específicas para avaliar aplicativos móveis educacionais. Esta pesquisa enfatizou o público infantil por meio de quatro etapas metodológicas.

A primeira etapa teve como objetivo investigar na literatura elementos caracterizadores do domínio infantil (ECDI), considerando o uso de tecnologias digitais no processo de aprendizagem das crianças. Para isso foram pesquisados artigos científicos por meio da plataforma Google Scholar ${ }^{5}$ com os seguintes termos: "elementos caracterizadores do domínio educacional infantil”, “jogos educacionais infantis", "jogos educacionais domínio infantil”, "características educacionais domínio infantil", "elements that characterize the children's educational domain", "educational characteristics for children", "children's educational games", "educational games in the children's domain".

Foram encontrados dois artigos (AN et al., 2013; GOMES et al., 2015) que propiciaram a fundamentação teórica necessária para a segunda etapa da pesquisa, ou seja, a partir desses estudos foi possível identificar os ECDI.

Na segunda etapa, os ECDI identificados em An et al. (2013) e Gomes et al. (2015) foram selecionados e classificados. Por meio da análise crítica, construiu-se um conjunto harmonizado de heurísticas com base nos ECDI que serviram de entrada para a terceira etapa. $\mathrm{Na}$ terceira etapa foi realizada a comparação entre as heurísticas de usabilidade para aplicativos móveis educacionais propostas por D'Carlo et al. (2016) com o conjunto harmonizado de heurísticas resultante da segunda etapa. Por meio dessa comparação foi possível propor, adaptar e

${ }^{5}$ https://scholar.google.com.br/ 
reutilizar heurísticas de usabilidade contidas no conjunto das HG que consideram os ECDI.

Em seguida, realizou-se a avaliação inicial do conjunto de heurísticas de usabilidade para aplicativos móveis educacionais infantis (quarta etapa) por especialista e por meio de um estudo comparativo experimental.

$\mathrm{Na}$ etapa de avaliação por especialistas, foi desenvolvido um questionário, com base em D'Carlo et al. (2016), para avaliar a relevância das heurísticas a partir da seguinte escala: Fortemente Relevante, Relevante, Pouco Relevante e Irrelevante. Esse questionário foi respondido por seis especialistas, pesquisadores e profissionais de IHC/Informática na Educação e da Educação Infantil. Após aplicação do questionário, realizou-se entrevista semiestruturada com cada um dos especialistas, com o objetivo de entender o motivo da relevância atribuída a cada heurística.

\subsection{Comparação das Heurísticas}

O estudo comparativo experimental baseou-se em D'Carlo et al. (2017). Nesse contexto, foi planejada a comparação entre as HE propostas e as HG. Para classificar a usabilidade do aplicativo foram utilizados critérios criados por Knoll (2014) e apresentados na Tabela 2. Segundo Nielsen (1994), a não detecção de uma violação heurística não atesta que ela não exista.

\section{Tabela 2 - Critérios de classificação de usabilidade}

\begin{tabular}{l|c} 
\% de heurísticas violadas & Classificação \\
\hline Menor ou igual a 25\% do total & Muito boa \\
Maior que 25\% e menor ou igual a 50\% & Boa \\
Maior que 50\% e menor que 75\% & Ruim \\
Maior ou igual a 75\% & Péssima
\end{tabular}

Fonte: Retirado de Knoll (2014).

A escolha do aplicativo móvel a ser experimentalmente avaliado baseou-se nos seguintes critérios: (1) aplicativo para plataforma Android@); (2) estar na categoria Educacional do Google Playß); (3) ser educacional; (4) destinado ao público infantil; (5) em português; (6) ser avaliado por pelo menos 75 mil usuários; (7) ter nota entre 4,0 e 5,0 na avaliação total de usuários do Google Playß; (8) ter um número de downloads superior a 10 milhões.

A partir desses critérios foi selecionado o aplicativo Tabuada de multiplicar ${ }^{6}$ (cf. Figura 1). Esse aplicativo é um quiz matemático de natureza educacional, destinado à aprendizagem e desenvolvimento do raciocínio lógico-matemático por crianças. O aplicativo possui quatro modalidades: aventura, estudo, desafio matemático e d uelo. Nessa última m odalidade, duas crianças podem jogar simultaneamente em um único dispositivo móvel. Todas as modalidades treinam as habilidades das crianças em operações de multiplicação.

Nielsen (1994) recomenda que para a avaliação heurística de usabilidade participem entre três e cinco especialistas em IHC. Nesse contexto, para participar deste experimento, 
Figura 1 - Aplicativo Educacional Infantil Tabuada de Multiplicar
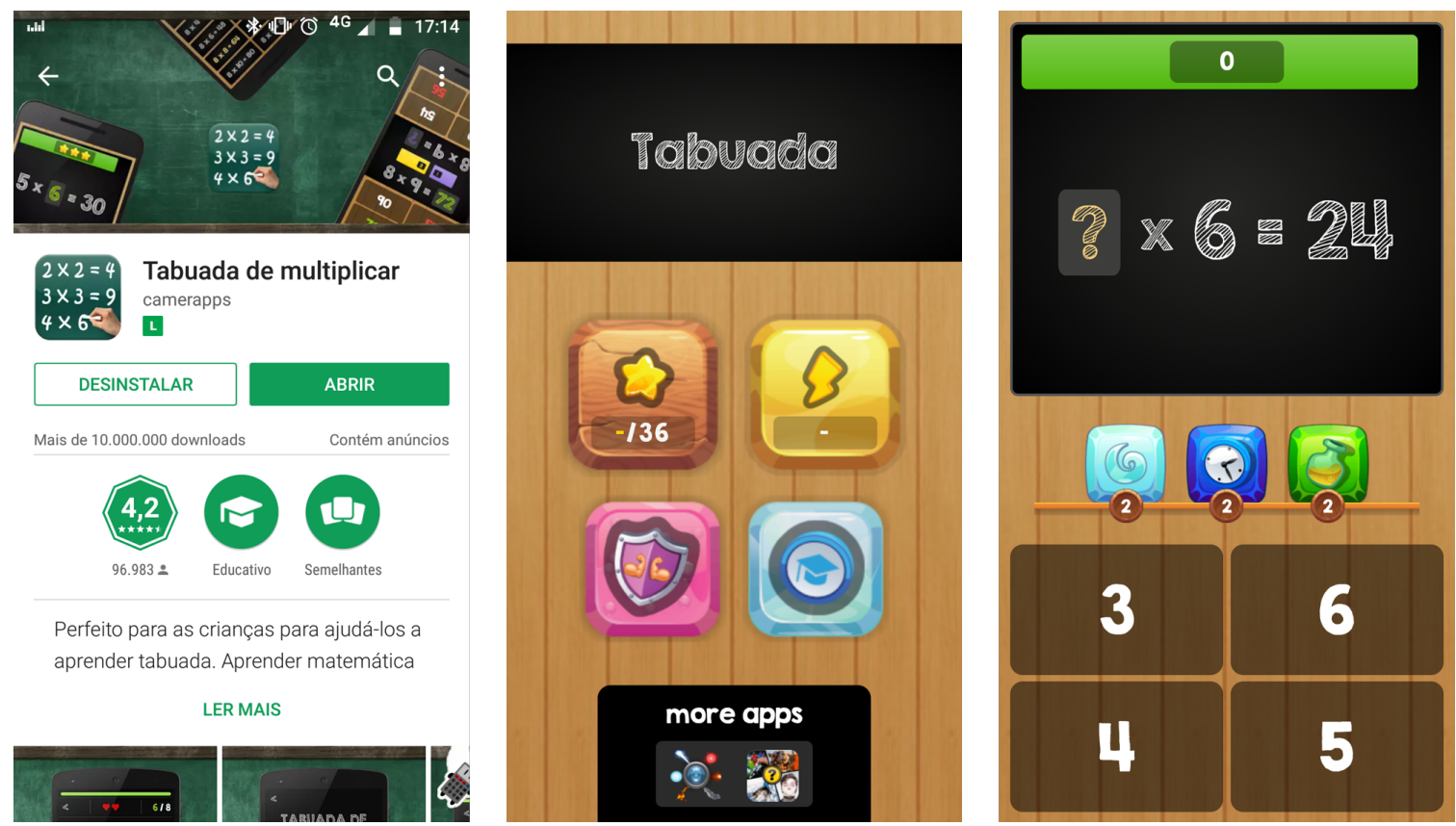

Fonte: Elaborado pelos autores.

foram convidados seis estudantes de pós-graduação em Ciência da Computação da linha de pesquisa em IHC (dois de doutorado e quatro de mestrado).

Os avaliadores inicialmente responderam um questionário, baseado em Bonifácio (2012), para identificar seus conhecimentos e experiência com IHC e avaliação heurística de usabilidade (AH). O resultado do questionário foi utilizado para organizar os especialistas em dois grupos balanceados. Um grupo ficou responsável por utilizar as HG durante a inspeção e o outro grupo ficou responsável pelo uso das HE.

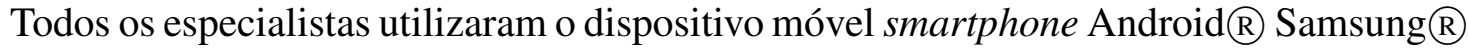

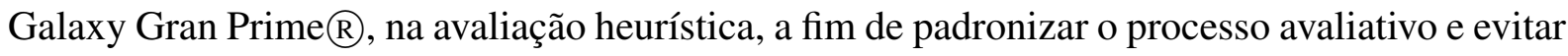
eventuais riscos ou limitações que poderiam surgir ao utilizar dispositivos diferentes. A inspeção do aplicativo seguiu a orientação de Nielsen (1994), na qual, separadamente, os avaliadores efetuaram reconhecimento do aplicativo Tabuada de multiplicar; cada avaliador de acordo com as respectivas heurísticas do seu grupo descreveu os problemas encontrados; indicou as heurísticas violadas; apontou o contexto onde ocorreu a violação; e, por fim, descreveu soluções propostas ou eventuais considerações.

\section{ELEMENTOS CARACTERIZADORES DO DOMÍNIO INFANTIL (ECDI)}

Esta seção é dedicada a apresentar os elementos caracterizadores do domínio educacional infantil, baseado em Gomes et al. (2015) e em An et al. (2013), respectivamente nas subseções 5.1 e 5.2 . 


\subsection{Elementos apresentados por Gomes et al. (2015)}

Após a realização da pesquisa bibliográfica para encontrar artigos relacionados aos ECDI, alguns resultados se mostraram interessantes, especialmente para o desenvolvimento do conjunto de heurísticas de usabilidade no domínio educacional infantil. Por exemplo, o trabalho de Gomes et al. (2015) apresenta 10 critérios para avaliar jogos educacionais infantis. Esses critérios serão nomeados aqui como "Parte 1" para facilitar as referências durante o processo de apreciação crítica desses critérios, apresentados a seguir:

Facilidade de uso: analisa a interação do usuário com o sistema e o número de erros cometidos durante essa interação;

Design da tela: analisa a qualidade do layout, considerando, por exemplo, se o jogo educacional apresenta fontes de fácil reconhecimento;

Apresentação da informação: facilidade de encontrar as informações básicas, próximas ações, ajuda, configurações e objetivos;

Afetividade: considera se o jogo proporciona um ambiente agradável, com elementos conectados ao mundo infantil, que possam criar laços emocionais entre os usuários/estudantes e o jogo;

Motivação: potencial de motivar o interesse, vontade, desejo e esforço dos usuários;

Objetividade: analisa a maneira na qual são dispostas as metas do jogo ao usuário, bem como a apresentação geral do software;

Papel do instrutor: observa o papel do instrutor no uso do jogo educacional, se provedor de materiais ou agente facilitador. No primeiro caso, o instrutor é considerado o detentor do conhecimento, enquanto no segundo ele e visto como uma fonte de orientação e consulta;

Estruturação: o jogo pode ser classificado como altamente estruturado, quando sua sequência/seus caminhos já foram determinados previamente; ou com baixa estruturação, quando o usuário pode escolher a ordem que desejar para seguir ao longo do jogo;

Controle do aluno: avalia a possibilidade do usuário controlar o encadeamento e realização das ações;

Aprendizado cooperativo: verifica se o jogo estimula o trabalho em equipe e a cooperação em algumas partes ou de forma integral, fazendo com que os objetivos possam ser compartilhados e os desafios divididos em uma equipe.

Destacam-se nos 10 critérios apresentados por Gomes et al. (2015) a afetividade, o papel do instrutor e o aprendizado cooperativo. A afetividade é observada com base na agradabilidade do jogo, com elementos conectados ao mundo infantil, que possam criar laços emocionais entre os alunos e o jogo. Esse item é importante especialmente no domínio infantil, devido às emoções que as crianças compartilham com os personagens do jogo e com o próprio ambiente. Segundo Amorim e Navarro (2012)

\footnotetext{
"a afetividade é necessária para a formação de pessoas felizes, seguras e capazes de conviver com o mundo a cerca, pois ela é uma importante aliada nas intenções pedagógicas, responsável por criar vínculos relevantes e imprescin-
} 
díveis para o Ensino de Educação Infantil [...]” (AMORIM; NAVARRO, 2012, p. 2).

Outro critério que merece destaque é o papel do instrutor. Nesse sentido, observa-se o papel do instrutor no uso do jogo educacional, se provedor de materiais ou agente facilitador. Este item é imprescindível ao processo de aprendizagem infantil, visto que professores nesse nível escolar procuram utilizar materiais que promovem o raciocínio e o desenvolvimento cognitivo das crianças. Por outro lado, o professor pode tomar o papel de agente facilitador em situação nas quais esse posicionamento seja relevante.

Por fim, evidencia-se o aprendizado cooperativo, que foi considerado como contido na heurística de usabilidade pedagógica "aprendizagem cooperativa/colaborativa" apresentada por Nokelainen (2006).

\subsection{Elementos apresentados por An et al. (2013)}

An et al. (2013) apresentaram um questionário destacando requisitos de usabilidade e requisitos pedagógicos de jogos digitais voltados ao público infantil. Esse questionário será nomeado "Parte 2" com intuito de facilitar as referências durante o processo de apreciação crítica. A Tabela 3 é uma reconstrução do questionário apresentado por An et al. (2013).

\section{Tabela 3 - Requisitos pedagógicos e de usabilidade no domínio educacional infantil}

\section{REQUISITOS}

\section{Requisitos de Usabilidade}

1. A linguagem é adequada ao público-alvo definido e ao nível de ensino a que se refere.

2. O jogo é atrativo envolvendo e cativando o aluno em sua utilização. O espaço é explorado e são usadas multimídias.

3. O tema é apresentado de forma lúdica e explora uma metáfora esclarecedora para o aluno.

4. O jogo permite e incentiva a crescente autonomia e o envolvimento.

5. O aluno consegue interagir com o jogo facilmente. Existe interação com outros usuários. As interações são seguras e promovem os objetivos do jogo.

6. Promove a criatividade (podem existir vários caminhos/respostas/soluções).

7. O tempo de cada ação é adequado. Existe a possibilidade de repetição. As etapas não são exaustivas.

8. As regras do jogo são coerentes e elas estão de acordo com o mundo imaginário proposto pelo jogo.

9. O nível de concentração exigido está de acordo com público do jogo.

\section{Requisitos Pedagógicos}

10. O conteúdo é coerente e contextualizado com a área e o nível de ensino proposto.

11. O grau de dificuldade do programa é variável, podendo ser definido pelo aluno ou ao passar de níveis conforme com os avanços obtidos.

12. Aborda os conteúdos de forma a facilitar o aprendizado.

13. Oferece feedbacks construtivos permitindo ao aluno identificar claramente quando acertou e repensar suas ideias e estratégias quando não forem bem sucedidas. Possui possibilidade de autocorreção.

14. O número de pontos obtidos é computado e é dado algum incentivo ou premiação ao atingir alguns marcos a fim de motivar o aluno.

15. Faz referência ao universo cotidiano dos alunos em uma perspectiva de formação e de cidadania.

Fonte: Retirado de An et al. (2013).

Dentre os elementos apresentados por An et al. (2013), destaca-se o item 1. Esse item possui considerável relevância especialmente no contexto infantil, no qual a maturidade das crianças deve ser considerada e, portanto, a linguagem deve ser igualmente adequada. Com 
relação à ludicidade, aspecto considerado no item 3, Romera et al. (2007) destacam a sua importância no processo pedagógico da educação infantil e afirmam que "[...] o lúdico é conhecido dos profissionais que atuam na área da educação infantil, há unanimidade no reconhecimento de sua importância para o desenvolvimento da criança, assim como da necessidade que esta tem de vivenciá-lo" (p. 149).

Desse modo, a análise do uso de atividades lúdicas pode ser aplicada na avaliação de usabilidade de aplicativos móveis educacionais específicos ao domínio infantil, visto que esses aplicativos podem dar oferecer flexibilidade e agilidade aos professores no processo de ensino, como também elevarem a interatividade entre as crianças.

No tocante ao item 6, sobre criatividade e imaginação, Vygotsky (2004) fortalece o destaque a esse item afirmando que "a infância é considerada o momento em que a fantasia é altamente desenvolvida, e, de acordo com essa crença, conforme a criança se desenvolve, sua imaginação e a força de sua fantasia diminui” (p. 31 - tradução nossa).

Por outro lado, esse autor também afirma que "uma das áreas mais importantes da psicologia da criança e da educação é a questão da criatividade em crianças, o desenvolvimento dessa criatividade e sua importância para o desenvolvimento e maturação geral da criança" (VYGOTSKY, 2004, p. 11 - tradução nossa).

Por fim, ressalta-se o item 8, sobre as regras de jogo, Kishimoto (1994) declara que:

“[...] um sistema de regras permite identificar, em qualquer jogo, uma estrutura sequencial que especifica sua modalidade. [...] São estruturas sequenciais de regras que permitem diferenciar cada jogo, ocorrendo superposição com a situação lúdica, uma vez que, quando alguém joga, está executando regras do jogo e, ao mesmo tempo desenvolvendo uma atividade lúdica" (KISHIMOTO, 1994, p. 108).

Partindo dos critérios e requisitos, os quais caracterizam elementos relevantes ao domínio infantil, apresentados por Gomes et al. (2015), denominados "Parte 1" e, An et al. (2013), denominados "Parte 2", efetuada a apreciação crítica na "Parte 1" e na "Parte 2" foi possível criar o Tabela 1 com os elementos caracterizadores do domínio infantil.

\section{HEURÍSTICAS PARA APLICATIVOS MÓVEIS EDUCACIONAIS INFANTIS}

Esta seção apresenta o desenvolvimento e resultado da terceira etapa metodológica desta pesquisa. Com a proposta de se alcançar heurísticas de usabilidade para aplicativos móveis educacionais infantis, nesta etapa utilizou o conjunto de heurísticas de usabilidade para aplicativos móveis educacionais, proposto por D'Carlo et al. (2016) e os resultados da seção anterior, os quais apresentam um conjunto de heurísticas com base nos ECDI.

Todos os elementos de cada um dos conjuntos citados foram comparados. Essa comparação teve como finalidade combinar elementos semelhantes e remover elementos duplicados. Essa etapa resultou em um conjunto parcial de heurísticas de usabilidade para aplicativos móveis educacionais infantis. 


\section{Quadro 1 - Conjunto de heurísticas com base nos ECDI}

\begin{tabular}{|c|c|c|}
\hline Heurística & Descrição & Referência \\
\hline $\begin{array}{l}\text { 1. Facilidade } \\
\text { de Uso }\end{array}$ & $\begin{array}{l}\text { Diz respeito ao número de erros cometidos } \\
\text { durante a interação do usuário com o } \\
\text { sistema. }\end{array}$ & $\begin{array}{l}\text { Gomes et al. } \\
\quad(2015)\end{array}$ \\
\hline $\begin{array}{l}\text { 2. Design } \\
\text { da Tela }\end{array}$ & $\begin{array}{l}\text { Atenta à riqueza no layout, considerando } \\
\text { sua atratividade, fontes fáceis de reconhecer, } \\
\text { bom aproveitamento da tela, boa disposição } \\
\text { dos elementos e utilização de cores atraentes. }\end{array}$ & $\begin{array}{l}\text { Gomes et al. } \\
\quad(2015)\end{array}$ \\
\hline $\begin{array}{l}\text { 3. Apresentação } \\
\text { da Informação }\end{array}$ & $\begin{array}{l}\text { Facilidade de encontrar as informações básicas } \\
\text { do aplicativo como ajuda, configurações, } \\
\text { próximas ações, objetivos e documentação. }\end{array}$ & $\begin{array}{l}\text { Gomes et al. } \\
\text { (2015) }\end{array}$ \\
\hline 4. Afetividade & $\begin{array}{l}\text { Considera se o aplicativo proporciona um } \\
\text { ambiente agradavel, com elementos conectados } \\
\text { ao mundo infantil, que possam criar laços } \\
\text { emocionais entre os alunos e o aplicativo. }\end{array}$ & $\begin{array}{l}\text { Gomes et al. } \\
\quad(2015)\end{array}$ \\
\hline 5. Motivação & $\begin{array}{l}\text { Representa o potencial de motivar o interesse, } \\
\text { vontade, desejo e esforço dos alunos. }\end{array}$ & $\begin{array}{l}\text { Gomes et al. } \\
(2015)\end{array}$ \\
\hline 6. Objetividade & $\begin{array}{l}\text { Analisa a maneira na qual são dispostas as } \\
\text { metas do jogo ao usuário, bem como a } \\
\text { apresentação geral do aplicativo. }\end{array}$ & $\begin{array}{l}\text { Gomes et al. } \\
\quad(2015)\end{array}$ \\
\hline $\begin{array}{l}\text { 7. Papel do } \\
\text { Instrutor }\end{array}$ & $\begin{array}{l}\text { Observa o papel do instrutor no uso do } \\
\text { aplicativo educacional, se provedor de } \\
\text { materiais ou agente facilitador. }\end{array}$ & $\begin{array}{l}\text { Gomes et al. } \\
\quad(2015)\end{array}$ \\
\hline $\begin{array}{l}\text { 8. Controle } \\
\text { do Aluno }\end{array}$ & $\begin{array}{l}\text { Avalia a possibilidade do aluno controlar o } \\
\text { encadeamento e realização das ações. }\end{array}$ & $\begin{array}{l}\text { Gomes et al. } \\
\text { (2015) }\end{array}$ \\
\hline $\begin{array}{l}\text { 9. Aprendizado } \\
\text { Cooperativo }\end{array}$ & $\begin{array}{l}\text { Verifica se o aplicativo estimula o trabalho } \\
\text { em equipe e a cooperação em algumas partes } \\
\text { ou de forma integral, fazendo com que os } \\
\text { objetivos possam ser compartilhados e os } \\
\text { desafios divididos em uma equipe. }\end{array}$ & $\begin{array}{l}\text { Gomes et al. } \\
\quad(2015)\end{array}$ \\
\hline $\begin{array}{l}\text { 10. Linguagem } \\
\text { alvo }\end{array}$ & $\begin{array}{l}\text { A linguagem utilizada deve adequar-se ao } \\
\text { público-alvo e ao nível de ensino a que esse } \\
\text { público se refere. }\end{array}$ & $\begin{array}{l}\text { An et al. } \\
(2013)\end{array}$ \\
\hline 11. Entretenimento & $\begin{array}{l}\text { Utilização de múltiplas mídias, tais como: } \\
\text { imagens, animações, vídeos, músicas e sons } \\
\text { relacionados a elementos do aplicativo. }\end{array}$ & $\begin{array}{l}\text { An et al. } \\
(2013)\end{array}$ \\
\hline 12. Uso do Lúdico & $\begin{array}{l}\text { O aplicativo apresenta temas lúdicos e explora } \\
\text { a abordagem lúdica apresentada pelo tema } \\
\text { durante seu uso. }\end{array}$ & $\begin{array}{l}\text { An et al. } \\
(2013)\end{array}$ \\
\hline $\begin{array}{l}\text { 13. Criatividade } \\
\text { e Imaginação }\end{array}$ & $\begin{array}{l}\text { Existem diversos caminhos, respostas ou } \\
\text { soluções que auxiliam cognitivamente na } \\
\text { imaginação e criatividade do usuário. }\end{array}$ & $\begin{array}{l}\text { Ant et al. } \\
(2013)\end{array}$ \\
\hline $\begin{array}{l}\text { 14. Esforço } \\
\text { Cognitivo }\end{array}$ & $\begin{array}{l}\text { Avalia se a criança consegue realizar as ações } \\
\text { sem demandar um excesso de esforço cognitivo } \\
\text { (nível de concentração, memorização, raciocínio } \\
\text { lógico) e se este esforço está de acordo com o } \\
\text { público-alvo. }\end{array}$ & $\begin{array}{l}\text { An et al. } \\
(2013)\end{array}$ \\
\hline $\begin{array}{l}\text { 15. Sentido } \\
\text { Lógico }\end{array}$ & $\begin{array}{l}\text { O aplicativo apresenta regras implícitas ou } \\
\text { explícitas que norteiam o sentido lógico da } \\
\text { aplicação. }\end{array}$ & $\begin{array}{l}\text { An et al. } \\
(2013)\end{array}$ \\
\hline $\begin{array}{l}\text { 16. Coerência } \\
\text { no Conteúdo }\end{array}$ & $\begin{array}{l}\text { O conteúdo é coerente e contextualizado com a } \\
\text { área e o nível de ensino propostos. Ele deve } \\
\text { colaborar com a aprendizagem do público-alvo. }\end{array}$ & $\begin{array}{l}\text { An et al. } \\
(2013)\end{array}$ \\
\hline 17. Feedback & $\begin{array}{l}\text { Oferece feedback construtivo, permitindo ao } \\
\text { aluno identificar os erros e acertos, e repensar } \\
\text { suas ideias e estratégias quando não forem bem } \\
\text { sucedidas. O feedback deve ser agradável e não } \\
\text { constrangedor. }\end{array}$ & $\begin{array}{l}\text { An et al. } \\
\text { (2013) }\end{array}$ \\
\hline
\end{tabular}

Fonte: Elaborado pelos autores. 
Posteriormente, esse conjunto passou por uma etapa final de ajustes. Algumas heurísticas tiveram seus nomes e descrições ajustados, de modo a refletir as ECDI. O conjunto final de heurísticas de usabilidade para aplicativos móveis educacionais infantis está apresentado na Tabela 4.

\subsection{Avaliação das Heurísticas}

Os resultados obtidos por meio do questionário utilizado para avaliar a relevância das heurísticas propostas (Hn), indicaram percentual de aceitação de 81,5\%. Sendo que, deste percentual de aceitação, 54,5\% das heurísticas foram consideradas "Fortemente Relevantes" e 45,5\% como "Relevantes" ao contexto de aplicativos móveis educacionais infantis. A Gráfico 1 apresenta um gráfico de resultados que compara cada uma das heurísticas e seus percentuais de relevância conforme indicados no questionário.

\section{Gráfico 1 - Resultado das respostas do questionário}

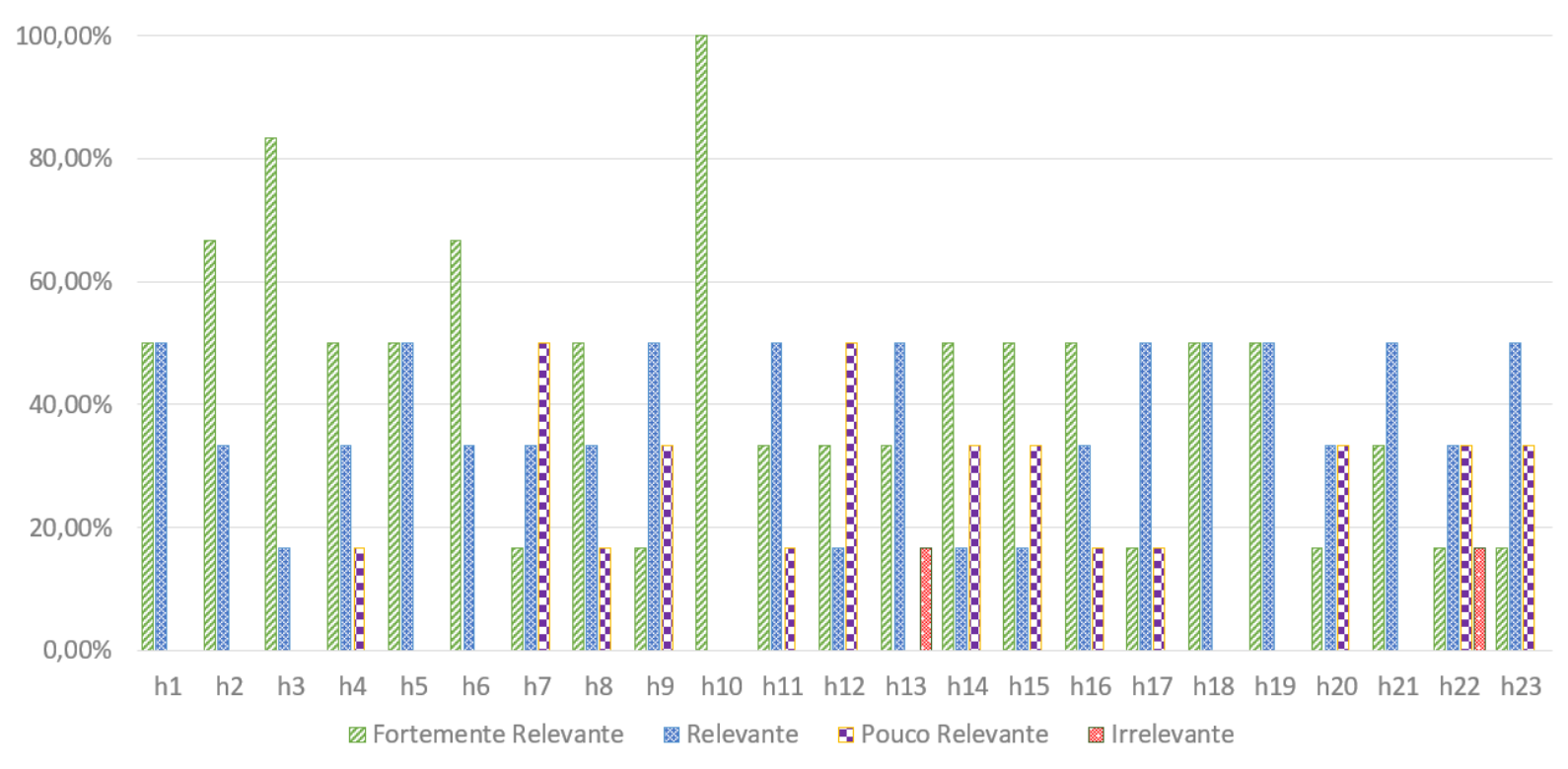

Fonte: Elaborado pelos autores.

Após a análise dos dados coletados por meio do questionário, foi realizada uma entrevista semiestruturada individualmente com cada especialista. Foi questionado aos participantes o motivo da escolha do grau de relevância para algumas das heurísticas que apresentaram divergência na avaliação. Essa etapa foi gravada e transcrita. Após a análise da transcrição, destacou-se algumas considerações concebidas por parte dos avaliadores. Seguem os dois destaques considerados mais relevantes pelos pesquisadores.

Um dos especialista indicou que apesar da importância da heurística "Heurística 7 (Papel do Instrutor)", a depender de como as informações são apresentadas no aplicativo, o papel do instrutor pode ser reduzido. Outro especialista reforçou a importância da "Heurística 14 (Esforço Cognitivo)" afirmando que um nível muito elevado pode despertar desinteresse na criança, referindo-se ao excesso de esforço cognitivo que é avaliado pela heurística. O especialista 


\section{Tabela 4 - Heurísticas para aplicativos móveis educacionais infantis}

\begin{tabular}{|c|c|}
\hline Heurística & Descrição \\
\hline $\begin{array}{l}\text { 1. Facilidade } \\
\text { de Uso }\end{array}$ & $\begin{array}{l}\text { Facilidade de acesso às funcionalidades. Medido através do número } \\
\text { de erros cometidos durante a interação do usuário com o sistema. }\end{array}$ \\
\hline $\begin{array}{l}\text { 2. Design da } \\
\text { Tela }\end{array}$ & $\begin{array}{l}\text { Atenta à riqueza no layout, considerando sua atratividade, fontes } \\
\text { fáceis de reconhecer, bom aproveitamento da tela, boa disposição } \\
\text { dos elementos e utilização de cores atraentes. }\end{array}$ \\
\hline $\begin{array}{l}\text { 3. Apresentação } \\
\text { da Informação }\end{array}$ & $\begin{array}{l}\text { Facilidade de encontrar as informações básicas do aplicativo como } \\
\text { ajuda, configurações, próximas açôes, objetivos e documentação. }\end{array}$ \\
\hline 4. Afetividade & $\begin{array}{l}\text { com elementos conectados ao mundo infantil, que possam criar } \\
\text { laços emocionais entre os usuários e o aplicativo. }\end{array}$ \\
\hline 5. Motivação & $\begin{array}{l}\text { Representa o potencial de motivar o interesse, vontade, desejo e } \\
\text { esforço do usuário. }\end{array}$ \\
\hline $\begin{array}{l}\text { 6. Aprendizado } \\
\text { Orientado a Metas } \\
\text { e Obietivos }\end{array}$ & $\begin{array}{l}\text { Analisa se o aplicativo possui metas e objetivos que colaboram com } \\
\text { o aprendizado do usuário. }\end{array}$ \\
\hline $\begin{array}{l}\text { 7. Papel do } \\
\text { Instrutor }\end{array}$ & $\begin{array}{l}\text { Observa o papel do instrutor no uso do aplicativo educacional, se } \\
\text { provedor de materiais ou agente facilitador. }\end{array}$ \\
\hline $\begin{array}{l}\text { 8. Controle do } \\
\text { Usuário }\end{array}$ & $\begin{array}{l}\text { Identifica se o usuário tem a liberdade de controlar as ações sobre o } \\
\text { software e os seus encadeamentos. }\end{array}$ \\
\hline $\begin{array}{l}\text { 9. Aprendizado } \\
\text { Cooperativo }\end{array}$ & $\begin{array}{l}\text { Verifica se o aplicativo estimula o trabalho em equipe e a } \\
\text { cooperação em algumas partes ou de forma integral, fazendo com } \\
\text { que os objetivos possam ser compartilhados e os desafios divididos } \\
\text { em uma equipe. }\end{array}$ \\
\hline $\begin{array}{l}\text { 10. Linguagem } \\
\text { alvo }\end{array}$ & $\begin{array}{l}\text { A linguagem utilizada deve adequar-se ao público-alvo e ao nível } \\
\text { de ensino a que esse público se refere. }\end{array}$ \\
\hline 11. Entretenimento & $\begin{array}{l}\text { Utilização de múltiplas mídias, tais como: imagens, animações, } \\
\text { vídeos, músicas e sons relacionados a elementos do aplicativo. }\end{array}$ \\
\hline 12. Uso do Lúdico & $\begin{array}{l}\text { O aplicativo apresenta temas lúdicos e explora a abordagem lúdica } \\
\text { apresentada pelo tema durante seu uso. }\end{array}$ \\
\hline $\begin{array}{l}\text { 13. Criatividade e } \\
\text { Imaginação }\end{array}$ & $\begin{array}{l}\text { Existem diversos caminhos, respostas ou soluções que auxiliam } \\
\text { cognitivamente na imaginação e criatividade do usuário. } \\
\text { Avalia se a criança consegue realizar as ações sem demandar um }\end{array}$ \\
\hline $\begin{array}{l}\text { 14. Esforço } \\
\text { Cognitivo }\end{array}$ & $\begin{array}{l}\text { excesso de esforço cognitivo (nível de concentração, memorização, } \\
\text { raciocínio lógico) e se este esforço está de acordo com o público } \\
\text { alvo. }\end{array}$ \\
\hline 15. Sentido Lógico & $\begin{array}{l}\text { O aplicativo apresenta regras implícitas ou explícitas que } \\
\text { norteiam o sentido lógico da aplicação. }\end{array}$ \\
\hline $\begin{array}{l}\text { 16. Coerência no } \\
\text { Conteúdo }\end{array}$ & $\begin{array}{l}\text { O conteúdo é coerente e contextualizado com a área e o nível de } \\
\text { ensino propostos. Ele deve colaborar com a aprendizagem do } \\
\text { público-alvo. }\end{array}$ \\
\hline $\begin{array}{l}\text { 17. Feedback } \\
\text { Construtivo }\end{array}$ & $\begin{array}{l}\text { Oferece feedback construtivo, permitindo ao usuário identificar } \\
\text { os erros e acertos, e repensar suas ideias e estratégias quando não } \\
\text { forem bem sucedidas. O feedback deve ser agradável e não } \\
\text { constrangedor. }\end{array}$ \\
\hline $\begin{array}{l}\text { 18. Disponibilidade } \\
\text { e Portabilidade }\end{array}$ & $\begin{array}{l}\text { O aplicativo deve fornecer acesso de qualquer lugar } \\
\text { (Disponibilidade) e deve ser capaz de ser executado em } \\
\text { diferentes sistemas e dispositivos móveis (Portabilidade). }\end{array}$ \\
\hline $\begin{array}{l}\text { 19. Resposta Imediata } \\
\text { e Visibilidade }\end{array}$ & $\begin{array}{l}\text { A aplicação deve fornecer uma resposta imediata à ação do } \\
\text { usuário. Esta resposta deve ser visível através da interface do } \\
\text { sistema. }\end{array}$ \\
\hline 20. Adequação de & \\
\hline $\begin{array}{l}\text { Mensagem à } \\
\text { Funcionalidade e } \\
\text { ao Usuário }\end{array}$ & $\begin{array}{l}\text { A mensagem deve se adequar às funcionalidades do sistema e às } \\
\text { ações tomadas pelo usuário. }\end{array}$ \\
\hline $\begin{array}{l}\text { 21. Prevenção de Erros } \\
\text { e Recuperação Rápida } \\
\text { do Sistema }\end{array}$ & $\begin{array}{l}\text { O sistema deve prevenir erros e ser capaz de oferecer uma rápida } \\
\text { recuperação ao último estado estável ou a um novo estado estável. }\end{array}$ \\
\hline $\begin{array}{l}\text { 22. Aprendizagem e } \\
\text { Recordação }\end{array}$ & $\begin{array}{l}\text { Avalia se o usuário é capaz de consolidar novos aprendizados } \\
\text { através do uso do aplicativo e recordar as ações e o conteúdo } \\
\text { apresentados durante o processo de interação. }\end{array}$ \\
\hline $\begin{array}{l}\text { 23. Diferentes } \\
\text { Meios de } \\
\text { Aprendizado }\end{array}$ & $\begin{array}{l}\text { O aplicativo fornece diferentes experimentações de aprendizado } \\
\text { para o usuário, incluindo o aprendizado via erros construtivos. }\end{array}$ \\
\hline
\end{tabular}

Fonte: Elaborado pelos autores. 
advertiu que, por outro lado, "[...] se tiver um pouco mais de esforço para que a criança consiga entender as coisas, é válido para o aprendizado". "Justamente quando está fácil demais é que não fica interessante”, complementa outro especialista.

$\mathrm{Na}$ “Heurística 20 (Adequação de Mensagem à Funcionalidade e ao Usuário)", os especialistas indicaram que, como o público é infantil, quanto mais intuitivo for o uso do aplicativo, mais fácil será para as crianças o utilizarem. Dessa maneira, quanto menos explicação o sistema tiver que apresentar, melhor será a utilização por parte das crianças. Essa afirmação sugere que mensagens mais curtas podem ser mais adequadas ao público infantil.

\section{AVALIAÇÃO DAS HEURÍSTICAS ESPECÍFICAS}

A partir da metodologia proposta, os grupos de especialistas avaliaram o software Tabuada de multiplicar, cada um com o seu respectivo conjunto de heurística. Os grupos encontraram 52 problemas ao total. A partir dos critérios apresentados por Knoll (2014), o aplicativo Tabuada de multiplicar foi classificado com "péssima usabilidade", tanto para as HG, quanto para as HE. A Tabela 5 apresenta os dados obtidos.

Tabela 5 - Classificação da usabilidade do aplicativo

\begin{tabular}{c|ccc} 
& $\begin{array}{c}\text { Qtde. de } \\
\text { Violações }\end{array}$ & $\begin{array}{c}\text { Heurísticas } \\
\text { Violadas }\end{array}$ & Classificação \\
\hline HG & 28 & $85,71 \%$ & Péssimo \\
HE & 60 & $91,30 \%$ & Péssimo
\end{tabular}

Fonte: Elaborado pelos autores.

Ao comparar os resultados apresentados na Tabela 5, verificou-se que as HE tiveram mais heurísticas violadas do que as HG. De acordo com os especialistas, algumas heurísticas foram violadas múltiplas vezes. Ao analisar a quantidade total de violações por conjunto (HE e $\mathrm{HG}$ ), concluiu-se que as HE conseguiram identificar um número de violações equivalente a mais que o dobro daquelas identificadas pelas HG. Esses dados indicam que as HE, portanto, possuem maior grau de cobertura para o contexto educacional infantil do que as HG.

As HE apresentaram uma média de violações superior as HG e equivalente a 2,86 violações por heurística violada. Parte considerável das HE, especialmente aquelas destinadas ao público infantil, se destacaram por terem detectado um volume de violações superior a média apresentada. Dentre essas heurísticas pode-se destacar a HE4 (Afetividade), HE10 (Linguagem alvo), HE14 (Esforço Cognitivo), H16 (Coerência no Conteúdo) e HE17 (Feedback Construtivo).

Ao fazer uma análise qualitativa das HE que ficaram acima da média de violações, os especialistas descreveram problemas e sugestões referentes a essas heurísticas. Em relação à HE4, os especialistas destacaram que "o aplicativo não propõe elementos conectados ao mundo infantil" e sugeriram considerar o uso de personagens nas telas do jogo. No tocante à heurística HE10, dois especialistas evidenciaram que a linguagem utilizada no aplicativo não é adequada para crianças e sugeriram o uso de uma linguagem mais simples. 
Em referência a HE14, um especialista indicou que "o aplicativo não possui um direcionamento lógico para o processo de aprendizado. Durante a interação o aluno deve primeiro interagir com a funcionalidade para saber o que é e para que serve a funcionalidade". Um segundo especialista ratificou essa informação ao afirmar que "existem 04 ícones e nenhuma descrição informando o que é, você só saberá se clicar e entrar. A heurística 14 é extremamente negligenciada nas telas deste aplicativo”.

Já no tocante a HE16, os especialistas evidenciaram a falta de coerência no conteúdo para crianças, visto que a tela inicial apresenta links/botões que não fazem parte do escopo do aplicativo. O especialista destacou ainda que esses links podem acarretar em erros para usuários, principalmente crianças e pessoas com deficiência. Também foi destacado o surgimento repentino de anúncios e propagandas totalmente incoerentes com o conteúdo destinado ao público infantil.

A HE17, por sua vez, foi a que teve o maior número de violações dentro do contexto educacional infantil. Um dos especialistas afirmou que "não é informado como funciona a pontuação e o feedback é muito limitado". Outro especialista evidenciou que o feedback não é construtivo e apenas indica o erro, sem explicação. Como solução foi indicado o uso de sons juntamente ao feedback; melhorar a percepção do erro; implementar uma forma de amarrar a pontuação ao progresso do usuário; armazenar os erros e indicar o que a criança deve revisar ao final do jogo.

Vale destacar que todas as heurísticas citadas anteriormente (HE4, HE10, HE14, HE16, HE17) são exclusivas do conjunto destinado a aplicativos móveis educacionais de uso infantil (HE) e suas violações não seriam detectadas por meio do conjunto genérico destinado a aplicativos móveis educacionais (HG).

\section{CONCLUSÃO}

Do ponto de vista científico, o conjunto de heurísticas poderá ser utilizado para avaliar a usabilidade de aplicativos móveis educacionais considerando características intrínsecas ao público infantil. Esse conjunto de heurísticas poderá ser explorado para avaliar aplicativos tanto em fase de projeto quanto aqueles já desenvolvidos. Acredita-se que na fase de projeto, essas heurísticas podem ser adaptadas a processos de design de interação, de modo a melhorar a qualidade da interação do aplicativo. Ao avaliar aplicativos já desenvolvidos, as heurísticas podem ser úteis ao redesign de interação e/ou para desenvolvimento de estratégias de marketing que evidencie a adequação do aplicativo ao público infantil. Desse modo, as heurísticas propostas pretendem aumentar a qualidade de interação entre usuário e sistema levando em consideração o domínio educacional infantil.

Além da contribuição científica, destaca-se o potencial benefício social, uma vez que a melhoria da usabilidade pode favorecer a busca por qualidade pedagógica das tecnologias educacionais atuais, especialmente os aplicativos móveis, a um novo patamar que compreenda 
as necessidades das crianças e melhore a qualidade de interação voltada a este público. Essas melhorias podem contribuir ainda para a inclusão social, uma vez que escolas e diversas instituições educacionais podem fazer uso desta pesquisa para imergir crianças de distintas realidades sociais em um meio educacional que contemple qualidade de interação e diversão.

Como trabalhos futuros, essas heurísticas serão avaliadas por meio de um estudo de caso com crianças, responsáveis e pedagogos ao utilizarem o aplicativo móvel educacional infantil Fantastic Pirates, apresentado por Abreu et al. (2017b). Posteriormente, o aplicativo descrito passará por uma Avaliação Heurística utilizando as heurísticas propostas por esta pesquisa.

\section{AGRADECIMENTOS}

O autor Jean C. S. Rosa agradece ao apoio da Coordenação de Aperfeiçoamento de Pessoal de Nível Superior (CAPES), por meio do programa CAPES-DS (\#88882.453913201901); e o autor Ecivaldo Matos agradece ao Conselho Nacional de Desenvolvimento Científico e Tecnológico - CNPq pela Bolsa de Produtividade em Pesquisa. 


\section{Referências}

ABREU, C. A.; ROSA, J. C. S.; MATOS, E. Aplicabilidade de heurísticas de usabilidade para aplicativos móveis educacionais infantis. In: SIMPÓSIO BRASILEIRO SOBRE FATORES HUMANOS EM SISTEMAS COMPUTACIONAIS, 16., 2017, Joinville. Anais [...]. Porto Alegre: SBC, 2017.

ABREU, C. A.; ROSA, J. C. S.; MATOS, E. Fantastic pirates: software de apoio ao ensino e à aprendizagem infantil. In: WORKSHOPS DO CONGRESSO BRASILEIRO DE INFORMÁTICA NA EDUCAÇÃO, 2017, Recife. Anais [...]. Porto Alegre: SBC, 2017.

ALMEIDA, R. . X. E. et al. Heurísticas de usabilidade orientadas às redes sociais. In: ENCONTRO DE ADMINISTRAÇAO DA INFORMAÇAO, 4., 2013, Bento Golçalves/RS. Anais [...]. Maringá: AnPAD, 2013. p. 1-15.

AMORIM, M. C. S.; NAVARRO, E. C. Afetividade na educação infantil. Interdisciplinar: Revista Eletrônica da Univar, Barra do Garças, v. 1, n. 7, p. 1-7, 2012.

AN, D. Y. et al. Digita-um jogo educativo de apoio ao processo de alfabetização infantil. In: SIMPÓSIO BRASILEIRO DE INFORMÁTICA NA EDUCAÇÃO, 24, 2013, Campinas. Anais [...]. Porto Alegre: SBC, 2013. p. 154-163.

ÁVILA, J.; MERINO, E. A. D.; MERINO, G. S. A. D. Usabilidade pedagógica: uma revisão sistemática da produção bibliográfica. Human Factors in Design, v. 6, n. 12, p. 124-143, 2012.

BONIFÁCIO, B.; OLIVEIRA, H.; CONTE, T. Avaliação de usabilidade de aplicações em dispositivos móveis. In: SYMPOSIUM ON HUMAN FACTORS IN COMPUTING SYSTEMS, 9., 2010, Belo Horizonte. Proccedings [...]. Porto Alegre: SBC, 2010. p. 269-270.

BONIFÁCIO, B. et al. Aplicando técnicas de inspeção de usabilidade para avaliar aplicações móveis. In: SYMPOSIUM ON HUMAN FACTORS IN COMPUTING SYSTEMS, 9., 2010, Belo Horizonte. Proccedings [...]. Porto Alegre: SBC, 2010. p. 189-192.

BONIFÁCIO, Bruno Araújo. Ubicua: uma técnica de inspeção de usabilidade para avaliar aplicações web móveis. 2012. Dissertação (Mestrado em Informática) - Universidade Federal do Amazonas, Manaus, 2012.

COELHO, O. B.; SANTORO, D. M. A sinergia entre as heurísticas de usabilidade de software e as heurísticas de ensino-aprendizagem do ponto de vista da educação à distância mediada pela web. In: SIMPÓSIO BRASILEIRO DE INFORMÁTICA NA EDUCAÇÃO, 13., 2002, São Leopoldo. Anais [...]. Porto Alegre: SBC, 2002. p. 273-281.

D'CARLO, D.; BARBOSA, G.; OLIVEIRA, E. Usabilidade em aplicativos móveis educacionais: Um conjunto de heurísticas para avaliação. In: SIMPÓSIO BRASILEIRO DE INFORMÁTICA NA EDUCAÇÃO, 27., 2016, Uberlândia. Anais [...]. Porto Alegre: SBC, 2016. p. 777-786.

D'CARLO, D.; BARBOSA, G.; OLIVEIRA, É. Proposta de um conjunto de heurísticas para avaliação da usabilidade de aplicativos móveis educacionais. Abakós, Belo Horizonte, n. 2, p. 16-35, 2017.

GOMES, A. S.; PADOVANI, S. Usabilidade no ciclo de desenvolvimento de software educativo. In: SIMPÓSIO BRASILEIRO DE INFORMÁTICA NA EDUCAÇÃO, 16., 2005, Juíz de Fora. Anais [...]. Porto Alegre: SBC, 2005. 
GOMES, T. et al. Avaliação de um jogo educativo para o desenvolvimento do pensamento computacional na educação infantil. In: CONGRESSO BRASILEIRO DE INFORMÁTICA NA EDUCAÇÃO, 4., 2015, Maceió. Anais [...]. Porto Alegre: SBC, 2015. p. 1349-1358.

GONÇALVES, V. P. et al. Uma revisão sistemática sobre métodos de avaliação de usabilidade aplicados em software de telefones celulares. In: BRAZILIAN SYMPOSIUM ON HUMAN FACTORS IN COMPUTING SYSTEMS, 10., AND THE LATIN AMERICAN CONFERENCE ON HUMAN-COMPUTER INTERACTION, 5., 2011, Porto de Galinhas. Proceedings [...]. Porto Alegre: SBC, 2011. p. 197-201.

KISHIMOTO, T. M. O jogo e a educação infantil. Perspectiva, Florianópolis, v. 12, n. 22, p. 105-128, 1994.

KNOLL, R. C. Desenvolvimento de heurísticas de usabilidade para tablets. Caderno de Estudos Tecnológicos, Bauru, v. 2, n. 1, p. 93-109, 2014.

MATOS, E. S. Dialética da Interação Humano-Computador: tratamento didático do diálogo midiatizado. 2013. Tese (Doutorado em Educação) - Universidade de São Paulo, São Paulo, 2013.

NIELSEN, J. Evaluating hypertext usability. In: JONASSEN, D. H. AND MANDL, H. Designing Hypermedia for Learning. Berlin, Heidelberg: Springer Berlin Heidelberg, 1990. p. $147-168$.

NIELSEN, J. Usability engineering. New York, USA: Morgan Kaufmann, 1994.

NOKELAINEN, P. An empirical assessment of pedagogical usability criteria for digital learning material with elementary school students. Journal of Educational Technology \& Society, v. 9, n. 2, 2006.

PICONEZ, S. C. B. Avaliação do potencial de usabilidade de material digital de aprendizagem: algumas contribuições. Série Educação e Tecnologia, 2010.

ROMERA, L. et al. O lúdico no processo pedagógico da educação infantil : importante, porém ausente. Movimento, Porto Alegre, v. 13, n. 2, p. 131-152, 2007.

SALAZAR, L. H. A. et al. Customizando heurísticas de usabilidade para celulares. In: 11TH BRAZILIAN SYMPOSIUM ON HUMAN FACTORS IN COMPUTING SYSTEMS, 2012, Cuiabá. Proceedings [...]. Porto Alegre: SBC, 2012. p. 37-38.

SCHUCK, S.; KEARNEY, M.; BURDEN, K. Exploring mobile learning in the third space. Technology, Pedagogy and Education, Routledge, v. 26, n. 2, p. 121-137, 2017.

SILVEIRA, M. S.; CARNEIRO, M. L. F. Diretrizes para a avaliação da usabilidade de objetos de aprendizagem. In: SIMPÓSIO BRASILEIRO DE INFORMÁTICA NA EDUCAÇÃO, 23., 2012, Rio de Janeiro. Anais [...]. Porto Alegre: SBC, 2012. p. 1-10.

SQUIRES, D.; PREECE, J. Usability and learning: Evaluating the potential of educational software. Computers and Education, v. 27, n. 1, p. 15-22, 1996.

VYGOTSKY, L. S. Imagination and creativity in childhood. Journal of Russian \& East European Psychology, Taylor \& Francis, v. 42, n. 1, p. 7-97, 2004.

WINCKLER, M.; PIMENTA, M. S. Avaliação de usabilidade de sites web. In: NEDEL, L. P. Escola de Informática da SBC SUL (ERI 2002). Porto Alegre: SBC, 2002. p. 85-137. 